\title{
HANET: A REMOTE SENSING AIRBORNE ARCHITECTURE FOR MULTI-FUNCTION ADHOC NETWORKS USING SDN.
}

\author{
Vikram Dhiman \\ I.K. Gujral Punjab Technical University, 144603, Distt. Kapurthala \\ vikramdhiman@lkcengg.edu.in \\ Manoj Kumar \\ DAVIT, Punjab 144001 India \\ drmanojkumarindia@gmail.com \\ Ajay K Sharma \\ I.K. Gujral Punjab Technical University, 144603, Distt. Kapurthala \\ vcptu13@gmail.com
}

\begin{abstract}
Proliferation and advancements for electronic wireless technologies have converged and motivated many researchers to evaluate and design a more scalable, reliable, and secure Ad-hoc network for ordinary civilians. Incorporating wireless technologies in a network always caters to human's life more conveniently, relaxing, and self-driven, where human intervention is challenging and overwhelming. However, available Ad-hoc networks are very rigid, limited to changes, and tough to manage, which doesn't allow flexibility, scalability, security, and performance in many aspects. To address said issues, we have proposed a new promising Ad-hoc network called HANET aim to support scalability and programmability. The primary goal of this model is to maintain the multi-domains state of technologies while acting as a middleware for applications such as battlefield, emergency search, and rescue, border patrol, surgical strike, agriculture monitoring, disaster warning, patient monitoring, and many more. The proposed paradigm targets to reduce the general cost for these applications and customize as per user convenience. The principle architecture and design characteristics of this paradigm have been discussed in the next session of this paper. This model motivates and contributes too many other aspects of the Adhoc network. This architecture incorporates the efforts of Airborne (AN) for route discovery and SDN to extended Ad-hoc networks from any corner.
\end{abstract}

Keywords: HANET, SDN, WSN, FUZZY, Ecosystem.

\section{INTRODUCTION}

In the era of sensors and wireless communication, terrestrial and airborne communication has become more potency. The evolution of SDN arises to a howling leap, helps defense and other private agencies in a cascade manner. SDN is the hope for any network to re-architect communication and methodology. We also treat the controller as a NOS network operating system. Hence can define the dynamics of the controller, which take precise information inside the network, for example, routes, bandwidth, jitter, and delay to provide a suitable path. Aspects of UAV [1] can be pinpoint as hunter-killer surveillance missions, commercial use, delivery of medicines in the battlefield, and aerial photography.

Similarly, the performance of the wireless sensor network and Adhoc network primarily depend upon the movement of nodes and its parameters associated with the longevity of the connection. One of the critical elements used to enhance the scalability and flexibility in the terrestrial and sky network is by using UAV or RPA drones, such as MQ-9 reaper, DJI Phantom, AltiGator, Tadiran Mastiff first flew in 1973[2]. In paper [3], author Yong Zeng proposed a new promising way to integrate $5 \mathrm{G}$ with the UAV network. This author added a tutorial review on areal UAV communication to address critical issues such as LOS dominant, GT [Ground Terminals] and Sky channel interference, quality of service, and SWAP [size, weight, and power] limitation in UAV device. In the desired process of tacking the inherent terrestrial challenges, Software Defined Networking has integrated with small UAVs. FANET recall that it is a particular form of MANET \& VANET[4] with high Mobility and low flight $\mathrm{z}$ autonomy. FANET, however, inherent problems like small flying duration and unproductive routing protocol[5]. However, since now, no adequate routing protocol has been proposed for such 
a network. Breakthrough in communication technologies has led to adopts Ad-hoc networks for many applications. Commercial domains required state of the art technologies to enhance quality, productivity, and precision in operations.

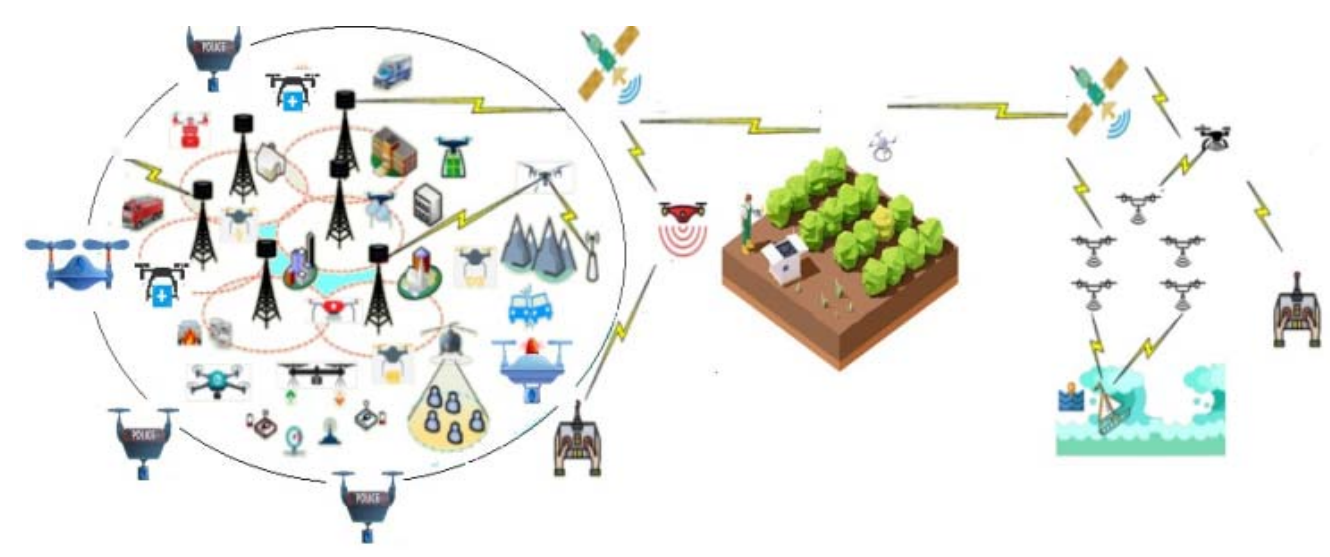

Figure 1.1 HANET Ecosystems, Networks for Advance Communication

For real-time communication and data gathering from the sensing field, using RPA is a promising and cemented solution for many problems. However, it also incorporates many inherent challenges, as discussed[6]. To maintain law and order, many countries such as the United States introduce the Federal Aviation Administration (FAA) for users to deploy autonomous aircraft, also must compliance under section 333 with a valid certificate of Authorization from the competent authority. Ideally, no positive regulation due to legal and liability Issues with RPA topology. Many software tools are emerging in the market for modeling and simulation to estimate performance. The software integrates many aspects required to simulate the algorithms and estimate the working of the various proposed protocols. In this paper, we have introduced a HANET (Hybrid Adhoc Network) designed by blending any network when demanded. The need for routing in the UAV network goes far from the scope of the VANET and MANET network.

\section{Network Open Issues in Adhoc Network}

MANET, VANET, WANET, FANET have open access issues to discuss before implementing HANET. In this category, we calm all the possible problems that have not been addressed with a promising solution for the designate network.

TABLE I OPEN ISSUES IN ADHOC NETWORK

\begin{tabular}{c|lll}
\hline $\begin{array}{c}\text { ADHOC } \\
\text { NETWORK }\end{array}$ & COMMUNICATION DEVICE & NETWORK LIMITATION & OPEN ISSUES \\
\hline MANET & Laptop & Mobility Problem & Merging existing \\
& Mobile & QoS & \\
& Palmtop & Hybrid connectivity & \\
& & Limited Mobility & \\
VANET & Vehicle2Vehicle & Standards & Merging existing \\
& communication & Hybrid connectivity & models \\
& & High Mobility & Merging existing \\
FANET & UAV (Unmanned Aerial & Standards & models. \\
& Vehicles) & Standard Mobility model & Limited to civil \\
& RPA (Remotely & Security & \\
& Piloted Aircraft) & QoS & \\
& & Standards & \\
& & Hybrid connectivity & \\
& & High Mobility & Adequate wireless \\
& & technology & \\
& & &
\end{tabular}




\begin{tabular}{|c|c|c|c|}
\hline WANET & $\begin{array}{l}\text { Desktop } \\
\text { Mobile device }\end{array}$ & $\begin{array}{l}\text { Hybrid connectivity } \\
\text { IPV6 capability. } \\
\text { Dynamic Routing protocols } \\
\text { Security }\end{array}$ & $\begin{array}{l}\text { Merging existing } \\
\text { models }\end{array}$ \\
\hline HANET & $\begin{array}{l}\text { Terrestrial to airborne Any } \\
\text { device with few } \\
\text { connectivity standards }\end{array}$ & $\begin{array}{l}\text { Standards } \\
\text { IPV6 capability. } \\
\text { LOS Reliability. } \\
\text { Dynamic Routing protocols } \\
\text { Middleware } \\
\text { MAC Protocol }\end{array}$ & $\begin{array}{l}\text { Smart AI } \\
\text { Controller } \\
\text { Resilience } \\
\text { Backup } \\
\text { connectivity in case } \\
\text { of system failure }\end{array}$ \\
\hline
\end{tabular}

\section{Motivation and Objective}

Motivation: 1) In India, scarcity in food supply for humans' survival, although $70 \%$ of the land has been cultivated and soon expected that till 2030 the population will be more than 1.5-2 billion people around the country that required advanced techniques for hassle-free farming. 2) A pandemic situation as Covid-19, we have seen a considerable life risk of Doctors, nurses, and medical staff to get engages near to the patient; using IOT based remote sensors, or robots it is possible to retrieve and perform the precise information and operation respectively to avoid such danger.

Objective: Our objective is to design a holistic Architecture and propose a route discovery protocol using FUZZY for multi-function Adhoc network, HANET ecosystems. This paper aim's at accomplishing the following:

1. HANET, Architecture.

2. SDN controller [open source].

3. Fuzzy, Approach.

It aims to address the applications trailing to the agriculture and medical field during a pandemic; this research serves art for Productive cultivation and precision monitoring, respectively.

\section{HANET Architecture}

UAV and sensor node plays a significant role in the deployment of such a model as both are used to serve the designate task.

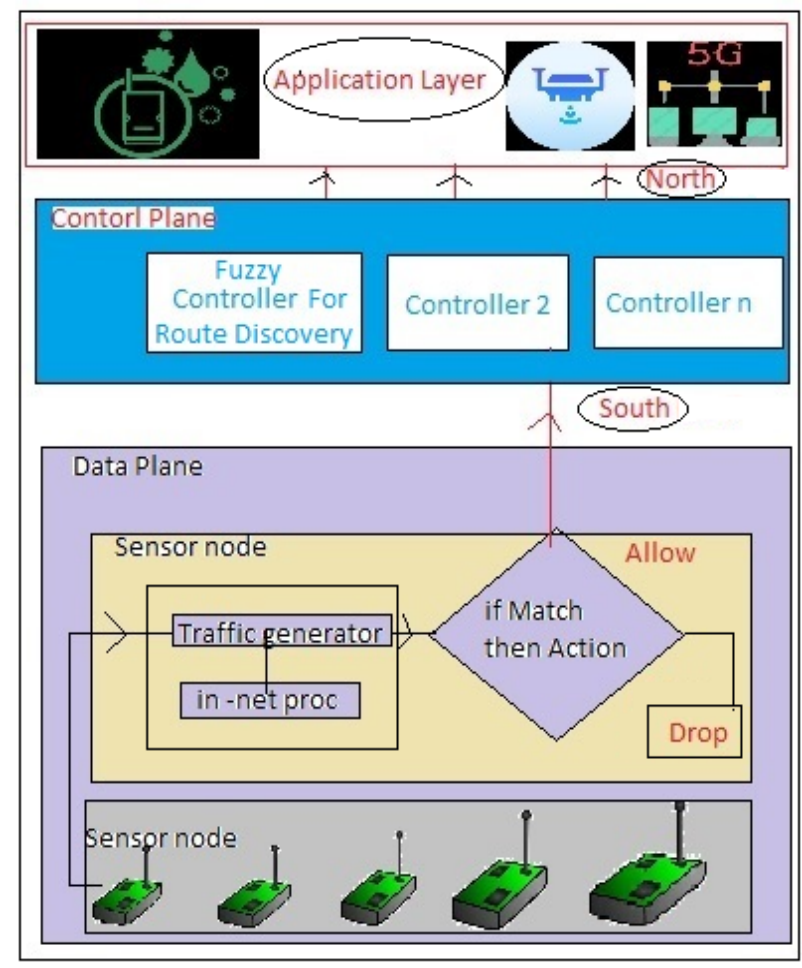

Figure 1.2 Architecture for Advance Communication in HANET 
Sensors are implemented to obtain the physical surrounding environment pertaining to applications, where UAVs have combined with WSN for better performance. UAV act as a mobile sink for transferring information more conveniently. Such a model helps in longevity as well as QoS in the sensor network. In addition to this, SDN is the emerging model to extend the existing working principle of any Adhoc network in a cascading manner.

\section{Literature Survey}

Y. B. Ko and N. H. Vaidya [7] introduced LAR consisting of two zones: request and expected. The proposed model results in high mitigation of routing overheads in the search area compared to algorithms not using LAR.

Hyunbum Kima, Jalel Ben-Othmanb, Paolo Bellavistac [8] introduced a framework for creating a collision-free reinforced barrier in the UAV network that involved the guarantee detection of intrusion. In addition to this author proposed a method to minimize the total movement distance of UAV using a zone-based novel approach.

D. Orfanus, E. Pignaton, and F. Eliassen[9] proposed a self-organizing paradigm for military UAV relay network; the UAV relay network incorporates networking with the backend $\mathrm{C} 2$ network. The objective is to maximized physical connectivity among UAVs, maximized the coverage, i.e., the distance among the UAV, and lastly, minimized the number of UAVs deployed. Bryan Kate JasonWaterman, Harvard University [10] proposed a simbeeotic framework gear towards modeling swarms of MAV (micro aerial vehicle), expound that simbeeotic incorporate an adequate level of fidelity to evaluate prototype model while maintaining the capability to test at scale.

In Paper [11], author Yi Wei examines dynamic mission planning in a multi-UAV network. In order to maintain the collaboration and coordination between multi-UAV, a new centralized controller is proposed based on DDDAS dynamics to the changing nature of the topology or environment. However, the central controller is similar to Base Station designed to the assigned task to UAV. To get the status inquiry, these central controllers send status Messages to the UAV device in a periodic interval. The central controller has a significant role element to play in this network.

In Paper PanGU[12], a software-defined network for wireless Sensor Network enables a centralized control while promising to preserve the flexibility and ad-hoc routing. Proposed routing is designed upon the OR opportunity routing stack. PanGU is developed to improve the performance of WSN and maintain the ad-hoc nature in the network.

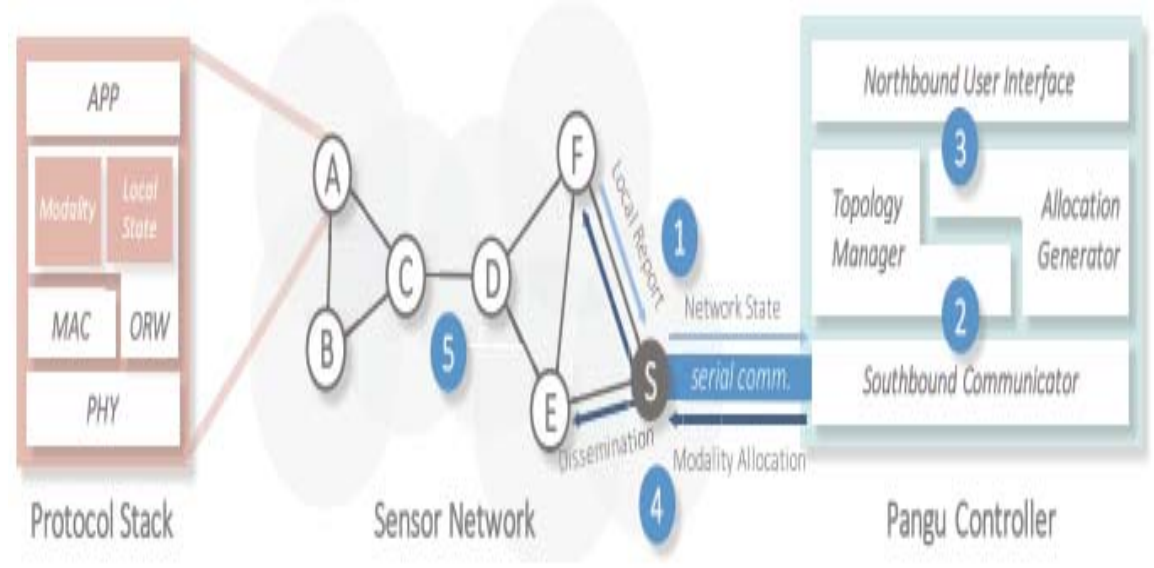

Figure 1.4 SDN architecture For WSN using PanGU[12]

In FUSN [1], author R. kirichek described a method of interaction between terrestrial and UAV networks to mitigate the difference between the ad-hoc characteristics of both terrestrial and UAV nodes. It was proposed to use the secondary nodes to overcome the routing functions using the SDN controller, where FUSN Flying ubiquitous sensor network-based Software Adhoc network is specially designed to reduce the total routing traffic. In this architecture, UAV-enabled SDN Based network management software-defined network controller is proposed.

A Fuzzy System Approach with QoE/QoS Guarantee [13] author Jorge Souza proposed a routing protocol based on the Fuzzy system with an object to discover high flight autonomy, low Mobility, and better RSSI. However, the level of Mobility is a concern with speed and depends on three linguistic values low speed (0 to $5 \mathrm{~m} / \mathrm{s}$ ), Average Speed (4 to $13 \mathrm{~m} / \mathrm{s}$ ), and High Speed greater than $11 \mathrm{~m} / \mathrm{s}$. similarly, other significant metrics is flight autonomy linked to battery divided into three linguistics level, ( 0 to $10 \mathrm{~min})$ low range, (10 to $20 \mathrm{~min})$ Average range, above 20 min High range. 
Whereas the last significant metric is signal quality denoted by RSSI (dbi) ranging to Low RSSI (-125.1 to $102.1 \mathrm{dbi})$, an average value is (-111.1 to $63.1 \mathrm{dbi}$,) and more significant than (-71.1 dbi) is higher RSSI. In this paper, the author proposed an adaptive routing protocol for FANET using fuzzy logic and later compared with AODV and OLSR protocols, using Gaussian Fuzzifier to reduce the input variable[13]. This type of interaction, as shown in figure 1.5, explains that the need for UAV for WSN, where approachability not feasible. Results showcase that the FANET adaptive routing protocol performed almost 300time better than AODV and OLSR protocols.

In paper [14], author A. Kakamoukas et al. examine the extensive review of Flying ad-hoc routing protocols and analytical study of available routing protocols best suites in agriculture applications for farmers to customized inputs to produce quality and productive crops. However, these communications classified technology-based routing into three categories: 1) Proactive 2) Reactive 3) Hybrid, where Destination Sequenced Distance Vector (DSDV) is a proactive routing protocol (OLSR) is also proactive and table-driven based on Link State Routing (LSR) protocols. In paper [15], OLSR is more favorable to the application, where a long delay in transmitting the data packet would not be acceptable.

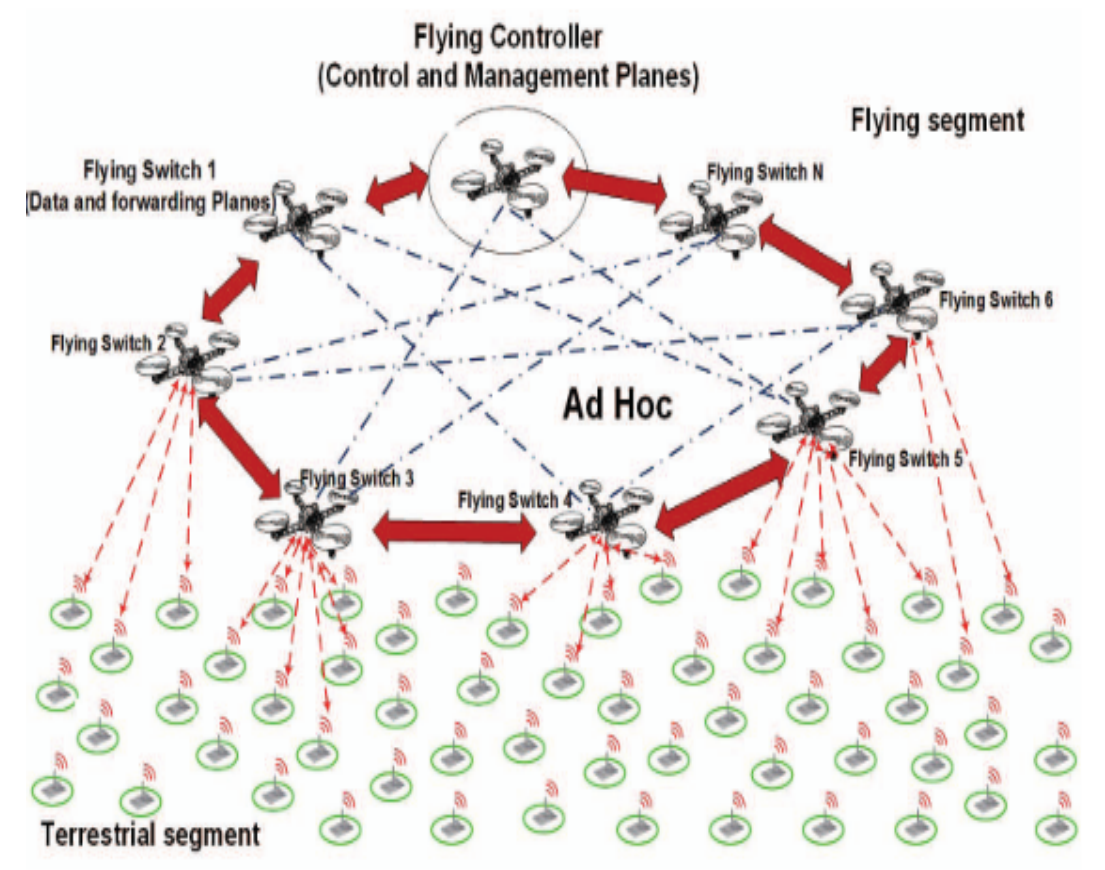

Figure 1.5 Interaction of Flying and Terrestrial Segments of FUSN[1]

The Dynamic Source Routing protocol (DSR)[16] is a simple and efficient reactive routing. Unlike DSDV and OLSR, DSR uses dynamic source routing with less overhead; it is a reactive/on-demand routing protocol, which means that the destination route can only be discovered when required; it consists of two-stage of working 1) Route Discover 2) Route maintenance although security is not addressed during DSR implementation. Another protocol in this category is AODV, with less overhead and easy adaption with less memory requirement. This protocol avoids loop routing due to 3 node instability and route poisoning. However, we have not found any algorithm that can promise higher data packet delivery with high Mobility, RSSI, and low flight autonomy.

In paper [17] author proposed the EAR new energy-aware routing and compared with AODV and DSR. However, the EAR result is receipted that the scheme is better in energy utilization since considering variable range transmission. The objective of MANET is to organize a network without any centralized controller so the node can act as both router and node at the same time; the author addresses the issue of energy efficiency to elevate the network's lifetime.

In Paper [18], Tie Luo et al. proposed a radical and composite approach to address and argue the issues inherent in the WSN network. The idea was to create the WSN network programmable using core component SOF [Sensor Open Flow].

In paper [19], author M.A. Araghizadeh et al. proposed the MAC protocol for the WSN-UAV network designed for emergency and monitoring applications. Conventional MAC protocols are not adequate for the unique design of such model UAV-WSN. However, the previous work was also missing the analytical evaluation of these protocols; this paper author designed a novel protocol called AP-MAC, using a Markov chain. It has also been receipted that the proposed protocol improves throughput and fairness about 20 and 25 percent, respectively. 
In paper [6], author Amira Chriki et al. discussed UAV Miniaturization and cost reduction for public use. Authors argue on the routing protocol, mobility optimization, and security model of UAV devices to give a general idea to the researchers.

Y. Qin, D. Huang, and B. Li, "STARS[2] In this paper, the author has proposed a (STARS) enabled with Eigen analysis to enhance precision to derive traffic patterns in MANETs. Qin et al. proposed this scheme as a pure passive and doesn't require any analyzer for manet transmission. Interestingly simulation result depicted that the method outperforms the previous approach, where the inference rule conceives the point to point and end-to-end traffic metric.

In paper [20], author Jesus et al. proposed three types of SDN based solutions in terms of their aspects. 1) Multiapplication. 2) Task distribution. 3) Energy optimization. Moreover, SDN removes the rigidity inherited by the traditional network. All of these aspects are possible due to the separation of data from the control plane in network devices. In order to communicate between data and control planes, open-flow is the standard protocol used to describe between them.

The controller is acting as a central point to take the status and configure the network when required. Open flow architecture has three main entries, as shown in figure1.7 open-flow switch, (data plane) external controller, and open-flow protocol. Other NOS Network Operating systems[21] and protocols used to create application and control networks [22] are discussed in Table II and Table III, respectively, since each has different features depends upon different programming language and architecture.

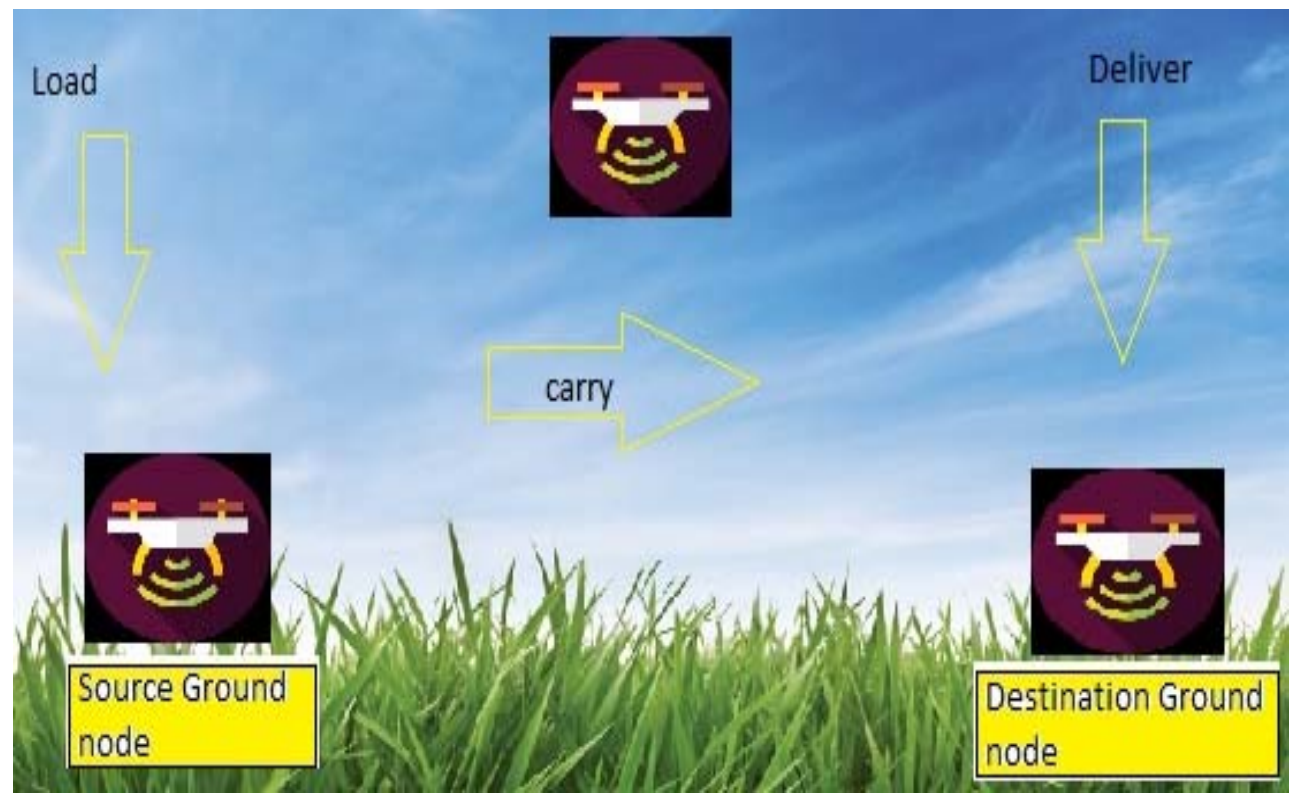

Figure 1.6 LCAD network with Three UAV's devices perform load, carry, and deliver three stages of communication[23].

In this paper[24], author Jose et al. proposed an energy-efficient solution using Unmanned Ground Vehicle for WSN. This approach includes event-triggered, packets based control, dual-rate controller, and Kalman filterbased prediction technique. These techniques help the UGV to achieve the desired path and hence saves the no of transmission from WSN which in turn lead to bandwidth and energy saving.

You can choose the controller according to the need for network and topology. The list of the controller is discussed in table III. However, we can invoke mininet, using simple CLI example \#mn, which stands mininet.

\$git clone git://github.com/osrg/RYU.git

\# mn --controller ref

\# mn --controller ovsc

\# mn --controller nox 
Table I Classification of Routing protocols for Adhoc Network

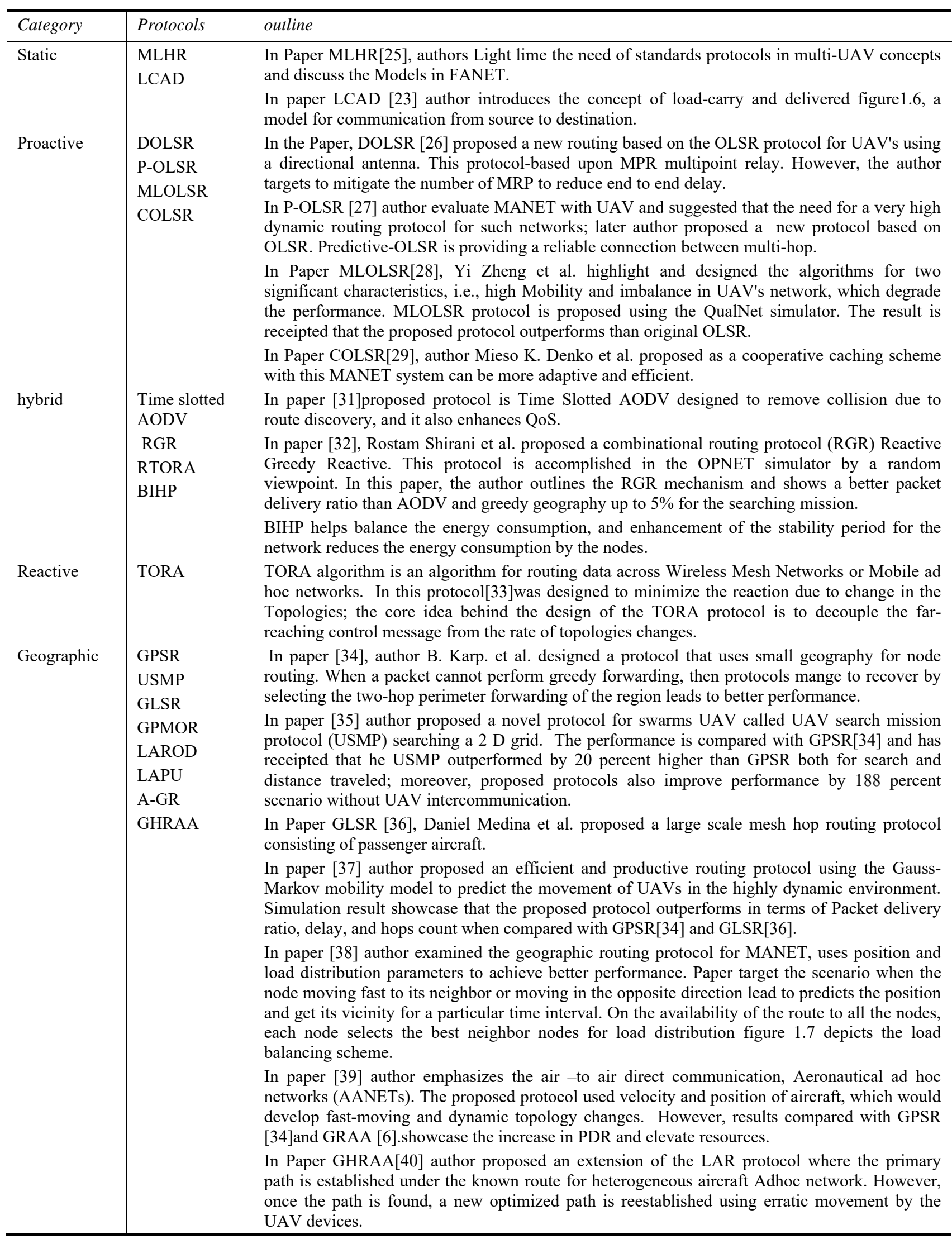


Sudo $\mathrm{mn}$-topo single, 3 -mac -switch ovsk - controller remote - $\mathrm{x}$.

Further to implement RYU, all the components are located and executed under/RYU/app directory with the support of switch using OpenFlow 1.3 version.

\#sudo mn -topo single, 3-mac-switch ovsk -controller remote - $\mathrm{x}$

RYU interacts as Figure 1.16 required switch and routers to redefine the network and modify, traffic flows. Each node has a unique ID and MAC address after deploying virtual SDN network topology using xterm windows.

Table III Types of NOS

\begin{tabular}{|c|c|c|c|}
\hline NOS Controller Name & Language type & Source & Platform Support \\
\hline $\operatorname{NOX}[41]$ & $\mathrm{C} / \mathrm{C}++$ & ICSI & Mostly Linux \\
\hline POX[21] & Python & ICSI & Any \\
\hline Maestro[42] & JAVA & RICE university & Any \\
\hline Beacon[43] & JAVA & Standford & Any \\
\hline Floodlight [44] & JAVA & Big Switch Network & Any \\
\hline ONOS[45] & JAVA & ON.Lab & Any \\
\hline OpenDay Light[46] & JAVA & Cisco and open-day light & Linux \\
\hline Frenetic [47] & OCaml & Princeton & Any \\
\hline Procera [48] & $\begin{array}{l}\text { Reactive policy } \\
\text { lang. }\end{array}$ & Microsoft research & windows \\
\hline Flowvisor proxy controller & $\mathrm{C}$ & Stanford & Any \\
\hline Terma & C/Ruby & NEC & Only Linux \\
\hline MUL SDN & $\mathrm{C} / \mathrm{C}++$ & Kulcloud & LINUX \\
\hline Mirage & OCaml & Cloud & Any \\
\hline Jaxon & Java & ID & Any \\
\hline RYU[49][50] & Python & NTT & Mostly Linux \\
\hline
\end{tabular}

\section{Design Characteristic and Frequency Range}

The Node Mobility, energy conservation, route routing, scalability, interoperability, communication, and security are the few key concern before designing any new paradigm.

In order to meet the design characteristic and to potentially address the inherent challenges in the Adhoc network, we have composed the SDN with the Adhoc network. However, the manual configuration of devices is not an adequate solution. The location-Aware application program interface allows the user to work in a weak system using a relocatable dynamic object, queued remote procedure, or RPC call. Scheduler, access manager, object cache, and operation log are the few components for rapid prototyping or Building such a network. Simulations can be conducted using a modified Simbeeotic[7] for fast prototyping simulation of MAV/UAV devices that can be implemented in a single scenario available to the community http://robobees.seas.harvard.edu, Simbeeotic is written in the Java programming language. It was designed \& developed as part of the RoboBees project at Harvard University, which provides a realistic physical model of small-sized UAVs, yielding crops in fields or patient health monitoring in hospitals [14]. EMU-Copter 17 persistently shares signal data to the base station for necessary action.

On the other hand, TOSSIM and Emstar are the two WSN simulators. Talking about current scenario applications is different from traditional networks; however, the fundamental principle is the same in the Adhoc network. 


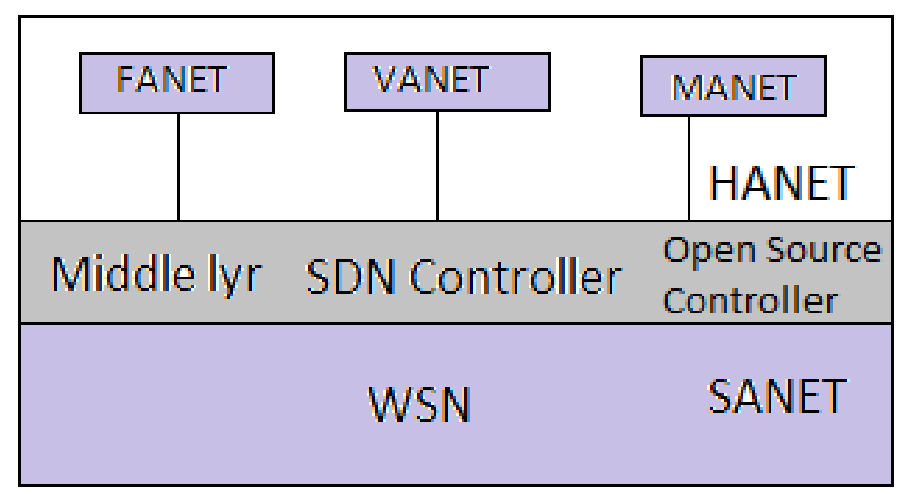

Figure1.7 Middleware SDN Controller

Current research works envisioned in enhancing the performance of the traditional network, addresses the issues, be solved in the future. In the same path, HANET is a novel way of connecting the ground station with Air Nodes. One of the biggest challenges us are agility, fidelity, and control of the network. In this study, we have discussed network architecture using the RYU controller. RYU is an open-source framework project designed by NTT fully implemented in python programming. In Japanese, it means flow, regarded as water god, which control rainfall and water bodies. RYU controller is designed to improve overall efficiency and reduce the cost of the entire network. It supports many protocols such as OpenFlow, Netconf, OVSDB, Of-config, many more. The union of RYU controller with Adhoc networks led to significant versatility to execute multiple applications using the same hardware. SDN based Adhoc network provides scalability, flexibility, and centralized control to achieve the best performance. RYU SDN controller has three layers named the application layer, middle layer, and control layer. The block structure of the RYU controller is shown in figure. Further, let's see how to sends a received packet to all the ports. A new method, 'packet_in_handler,' is added to the L2Switch class. This is called when Ryu receives an OpenFlow packet_in message. The trick is the 'set_ev_cls' decorator. This decorator tells Ryu when the decorated function should be called. The core component of the OpenFlow controller is 1) Handle connections from switches, 2) Generate and route events to appropriate entities like Ryu applications. Another reason for using a mininet simulator is that it, i.e., widely used for experimentation and provides customized topologies and full customization of packet forwarding inside the network. Using the iperf3 tool, we can evaluate the design structure of the Adhoc network for actual maximum available bandwidth. Its feature includes UDP and TCP bandwidth, delay, and jitter measures in the network. To get Results and demography from the Host 1 and Host 2, we use iperf tools since the command put the Results into a file called result. Used this result to plot graph with the help of GNU plot in mininet, by switching root

sudo $-\mathrm{i}$,

\# mn --topo=minimal

\#xterm h1 h2

mininet> h1 iperf -s -p 5566 -i $1 \&$

mininet $>$ h2 iperf $-\mathrm{c}$ 10.0.0.1 -p 5566 -t 100

\#iperf $-\mathrm{s}-\mathrm{c} 5566-\mathrm{i} 1>$ result

Before plotting the result into a graph, we need to install gunplot by \#apt-get install gnuplot $-\mathrm{x} 11$

gunplot> plot "result" title "flow tcp" with linespoints // will plot the result into a 2D graph.

SDN controller redefines the actual definition of networking in the sense of throughput, traffic management, load balancing, QoS, security, and Private networking without the use of Vlan's. In this paper, we apprehend the significance of SDN for today's era; table IV describes the frequency range and characteristics for the model proposed with the network. Software-Defined Network is an emerging technology designed with the RYU controller to address the optimal path and quality of services[51].In order to implement the SDN controller, simulator MiniNet[52] were best suited and easy to use. It uses namespace to create different network contexts for the individual thread running on a single machine. Open flow network allows direct program ability by the network; each device has API programmable. Once we have an API, we can have a layer of software above a controller. ONF (open network foundation) aims in the promotion and adaptation of the SDN network. OpenFlow is the first standard interface or gateway to communicate between the forward layer and control of SDN. This research became a project in 2007. To maintain consistency, we propose a dynamic network strategy to converge Adhoc network service based on SDN protocol and controller[53]. SDN uses an open flow protocol to collect prerequisites for communication such as topology discovery, route computing, and wireless connection status. 


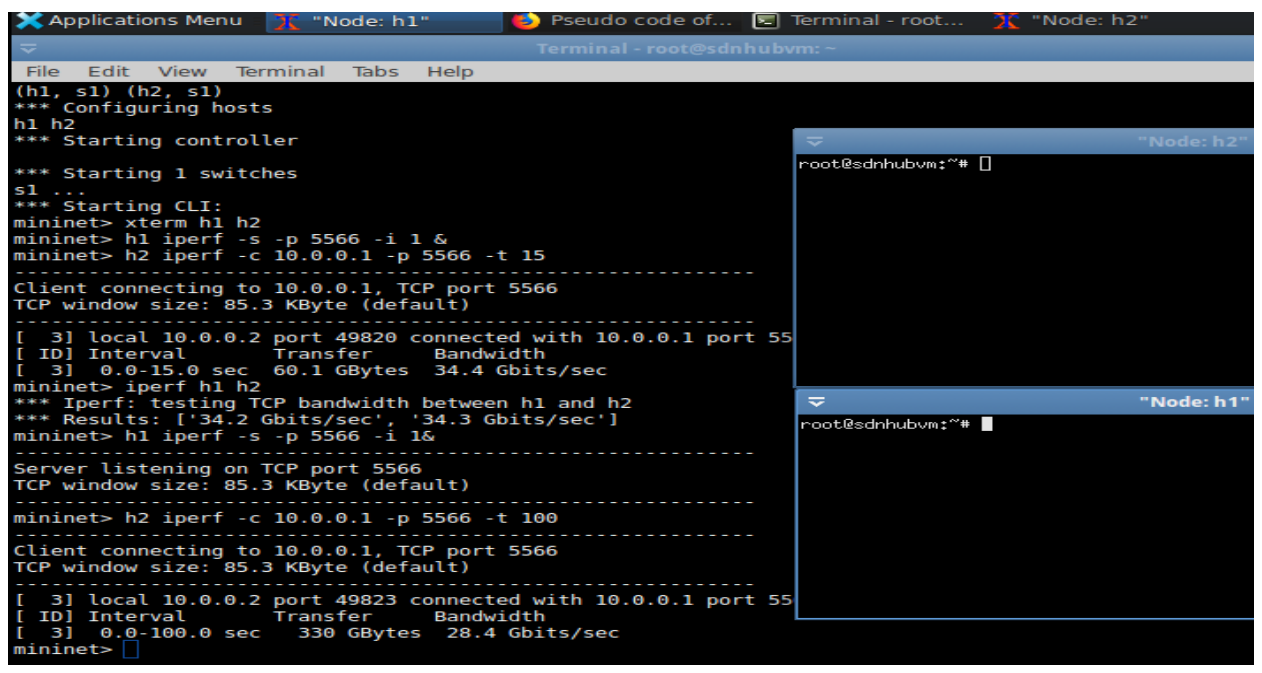

Figure1.8 Screenshot mininet iperf

All these parameters collect the required statistics and path for the network flow. However, a pragmatic implementation may hold many limitations and result in connection failure due to extreme topology change.

TABLE IV FREQUENCY RANGE

\begin{tabular}{c|lll}
$\begin{array}{c}\text { Band } \\
\text { Designation }\end{array}$ & Frequency Range & \multicolumn{1}{c}{ Usage } & Network \\
& $3-30 \mathrm{Mhz}$ & OTH surveillance & HANET/FANET \\
$\boldsymbol{V H F}$ & $30-300 \mathrm{Mhz}$ & Very long-range surveillance & FANET \\
\hline $\boldsymbol{U H F}$ & $300-1000 \mathrm{Mhz}$ & Very Long-range surveillance & HANET \\
$\boldsymbol{L}$ & $1-2 \mathrm{Ghz}$ & Long range surveillance & VANET \\
\hline $\boldsymbol{S}$ & $2-4 \mathrm{GHz}$ & Long-range weather & MANET \\
$\boldsymbol{C}$ & $4-8 \mathrm{GHz}$ & Long-range tracking & FANET \\
\hline $\boldsymbol{X}$ & $8-12 \mathrm{GHz}$ & Short-range tracking & HANET \\
$\boldsymbol{X}$ & $8-12 \mathrm{GHz}$ & Short-range tracking & HANET/ \\
\hline $\boldsymbol{K u}$ & $12-18 \mathrm{GHz}$ & High-resolution mapping & HANET \\
$\boldsymbol{K}$ & $18-27 \mathrm{GHz}$ & satellite altimetry & Little use \\
\hline $\boldsymbol{K a}$ & $27-40 \mathrm{GHz}$ & Very High range Airport & FANET/HANET \\
& & Surveillance & Little use
\end{tabular}

TABLE V ADHOC CHARACTERISTICS

\begin{tabular}{|c|c|c|c|c|}
\hline Characteristics & MANET & VANET & FANET & HANET \\
\hline Node Mobility & Less & Moderate & High & Extreme \\
\hline Node Density & Less & Moderate & Specific & High \\
\hline Topology change & less & Frequently & $\begin{array}{l}\text { Very } \\
\text { frequently }\end{array}$ & Extreme \\
\hline $\begin{array}{l}\text { Computational } \\
\text { Power }\end{array}$ & Less & More & High & Very High \\
\hline $\begin{array}{l}\text { Localization } \\
\text { Mobility Model }\end{array}$ & $\begin{array}{l}<100-200 \mathrm{~m} \\
\text { Odyssey } \\
\text { Client-Server }\end{array}$ & $\begin{array}{l}<300-400 \mathrm{~m} \\
\text { Odyssey } \\
\text { Client-Server }\end{array}$ & $\begin{array}{l}<900-1500 \mathrm{~m} \\
\text { Rover } \\
\text { Line of sight }\end{array}$ & $\begin{array}{l}>2000 \mathrm{~m} \\
\text { Rover Model } \\
\text { Relocatable } \\
\text { dynamic objects }\end{array}$ \\
\hline Network life Time & $\begin{array}{l}1-3 \text { working } \\
\text { hours }\end{array}$ & $\begin{array}{l}9-10 \text { working } \\
\text { hours }\end{array}$ & $\begin{array}{l}11-20 \text { working } \\
\text { hours }\end{array}$ & $>24$ \\
\hline
\end{tabular}


To achieve adequate management in UAV and make network competent SDN-UAV need to converge to form a high degree of coordination in the Adhoc network.

These devices can communicate using $802.16,802.11 \mathrm{a} / \mathrm{b} / \mathrm{g} / \mathrm{n}$, and 802.15.4. HANET network layer has to act adaptively between layers that correspond between traditional and modern networks [16]. Routing Hardware for Adhoc network required switches and the SDN controller; consequently, they play a vital role in overall architecture design. To improve efficiency, we separated the task of switches and controlling network using the data plane and control plane. They can decide the shortest path, filter traffic, route heavy traffic, data forwarding, packet drop, etc. Although sending the packet to the firewall in the switch led to filter the accept flow, it subsequently reduces the computation of the firewall for the rest of the packet as SDN controller and can bypass the firewall and removing unnecessary load and allowing gigabit data centers firewalled. SDN allows multiple flows to a different host by setting the initial flow's lead, reducing load balancing, and elevating the data rate of the network. It caters easily to creating a private network for an organization without actually spending in VLAN's. It also Inculcates TAP, sniffer for any port or traffic, allows programming the network by sampling the original stream of a traffic monitoring device.
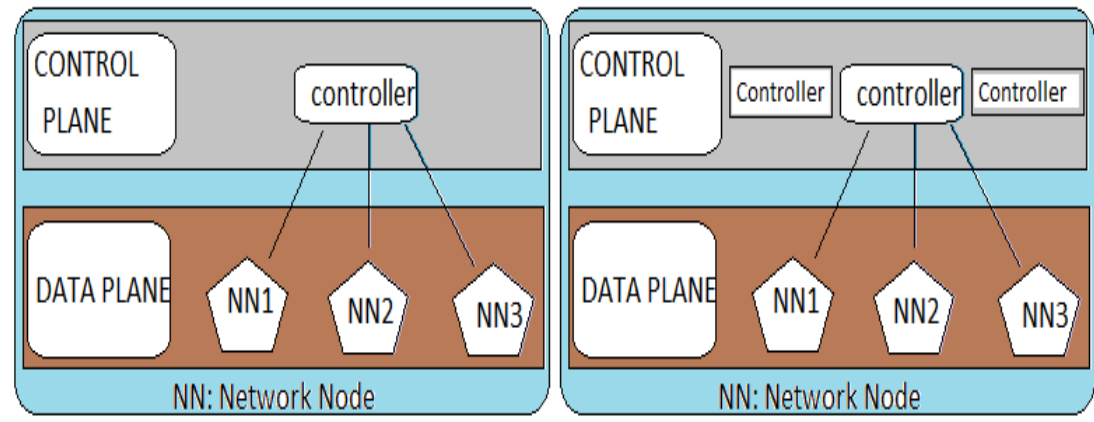

Figure 1.9 Single and Multi connected SDN Controller

\subsection{Network Assumptions:}

a) Here Sensors are deployed randomly using heterogeneous nodes.

b) All Sensor nodes have assigned a unique name, Ip address, and MAC address.

c) Communication between nodes took place using clustered chain routing and UAV.

d) Deploy four equipped controllers in a précised location, as shown in figure 1.10 [C1-C4].

e) SDN Controllers are GPS Enabled and know the UAV location consistently.

f) SDN Controllers are limitless, and failure of controller occurs due to power exhaustion.

g) Data delivery to the sink is carried out by UAV device, collecting data from the controller after receiving a signal (RTS) using directional antenna over predefined sliding portion SP1, SP2, and SP3 where $\mathrm{f}=60$ to avoid the hidden terminal problem.

h) UAV device starts their journey when both the controller [as shown in table VI] in the sliding path is ready to transmit to its UAV1 and similar to UAV2.
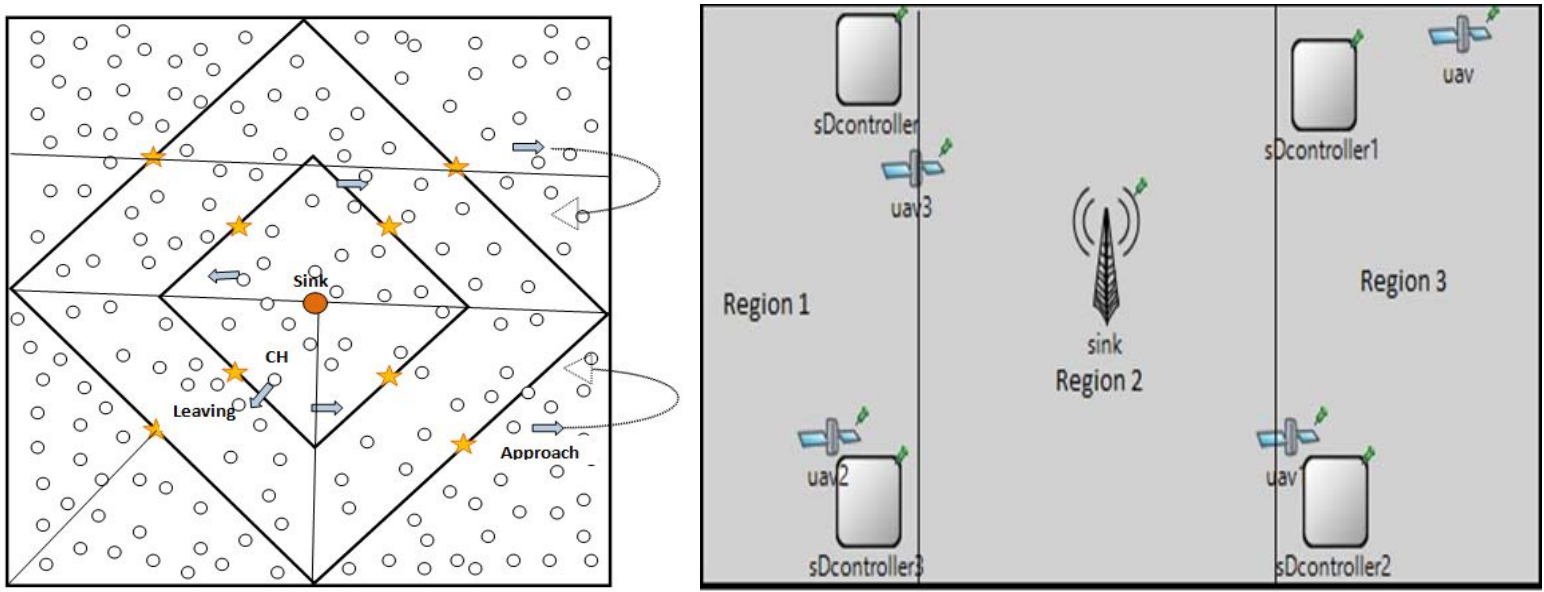

Figure1.10 HANET Network overview. 
The Energy consumption model described in our proposed work is based on Eqs(1) and Eqs(2).

$\mathrm{E}_{\mathrm{TX}}(\mathrm{d}, \mathrm{L})=\mathrm{L}^{*}$ Eelec $+\mathrm{L}^{*} \varepsilon_{\mathrm{fs}} \mathrm{d}^{2}$, if $\mathrm{d}<\mathrm{do}$

$\mathrm{E}_{\mathrm{TX}}(\mathrm{d}, \mathrm{L})=\mathrm{L} *$ Eelec $+\mathrm{L}^{*} \varepsilon_{\mathrm{mp}} \mathrm{d}^{4}$, if $\mathrm{d} \geq \mathrm{do}$

$\mathrm{E}_{\mathrm{RX}}(\mathrm{L})=\mathrm{L}$. Eelec

Whereas Energy Exhausted by Cluster Head. Given by Eqs(3).

$\mathrm{E}_{\mathrm{CH}}=(\mathrm{NET}) \mathrm{L}($ Eelec $+\varepsilon f s d B s+$ EDA $)$

The system design of the proposed model is a plan according to the environment while deploying the SD controller in the target region.

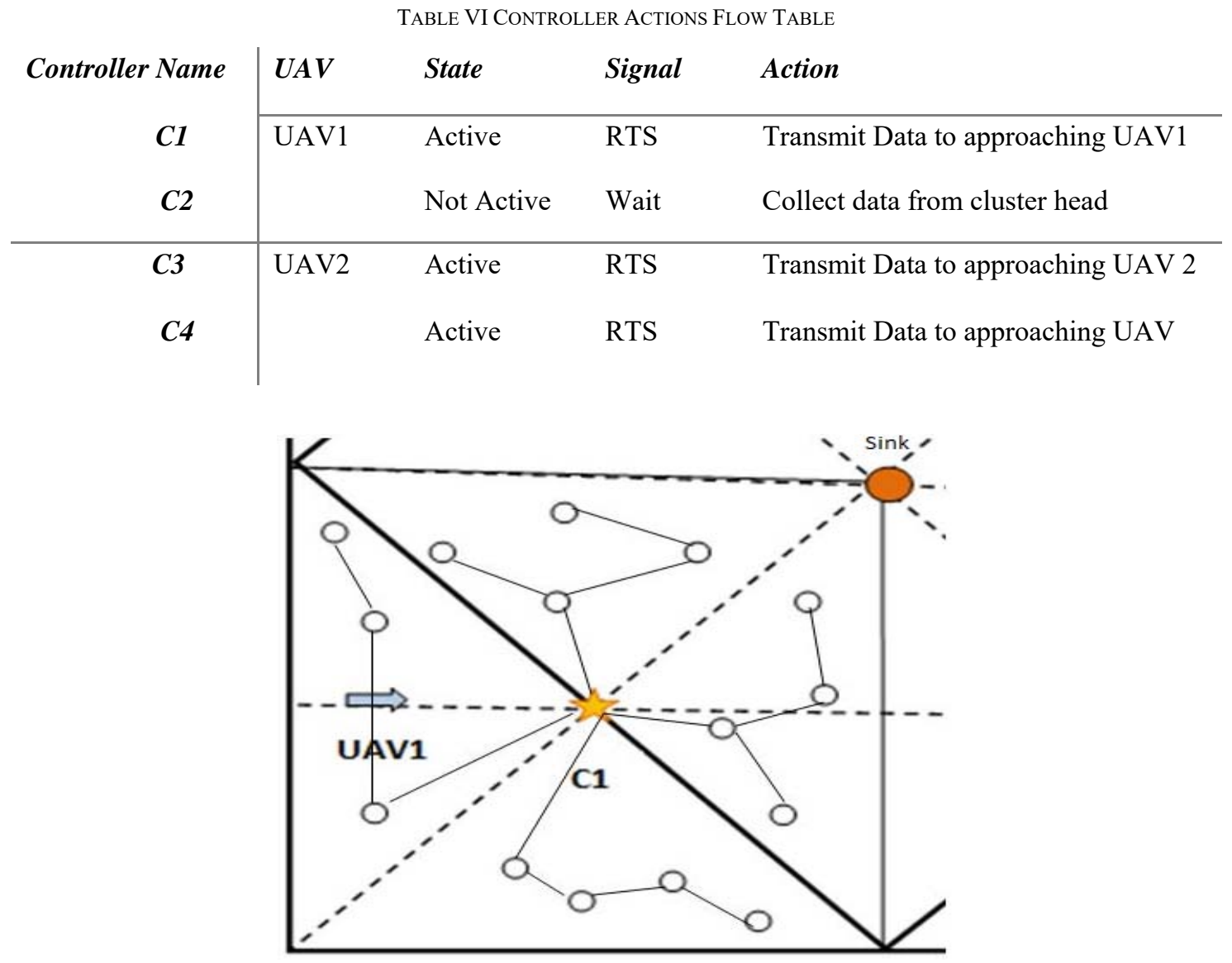

Figure1.11 UAV movement in Quradtor1

TABLE VI MULTi-UAV FunCtion

\begin{tabular}{|l|l|l|l|l|l|}
\hline UAV & $\begin{array}{l}\text { Current } \\
\text { location } \\
\text { GPS }\end{array}$ & $\begin{array}{l}\text { Residual } \\
\text { capacity in } \\
\%\end{array}$ & $\begin{array}{l}\text { The calculated } \\
\text { capacity } \\
\text { consumption rate }\end{array}$ & $\begin{array}{l}\text { Estimated } \\
\text { final location }\end{array}$ & $\begin{array}{l}\text { Estimated final residual } \\
\text { capacity after completing } \\
\text { all of its tasks }\end{array}$ \\
\hline Drone 1 & $\mathrm{X} 1, \mathrm{Y} 1$ & 90 & 20 & XFL1, YFL1 & 20 \\
\hline Drone 2 & $\mathrm{X} 2, \mathrm{Y} 2$ & 80 & 15 & XFL2, YFL2 & 60 \\
\hline Drone 3 & $\mathrm{X} 3, \mathrm{Y} 3$ & 100 & 10 & XFL3, YFL3 & 60 \\
\hline Drone $n-2$ & - & - & - & - & - \\
\hline- & - & - & - & - & - \\
\hline Drone $N$ & $\mathrm{Xn}, \mathrm{Yn}$ & 60 & 30 & XFLn, YFLn & 80 \\
\hline
\end{tabular}




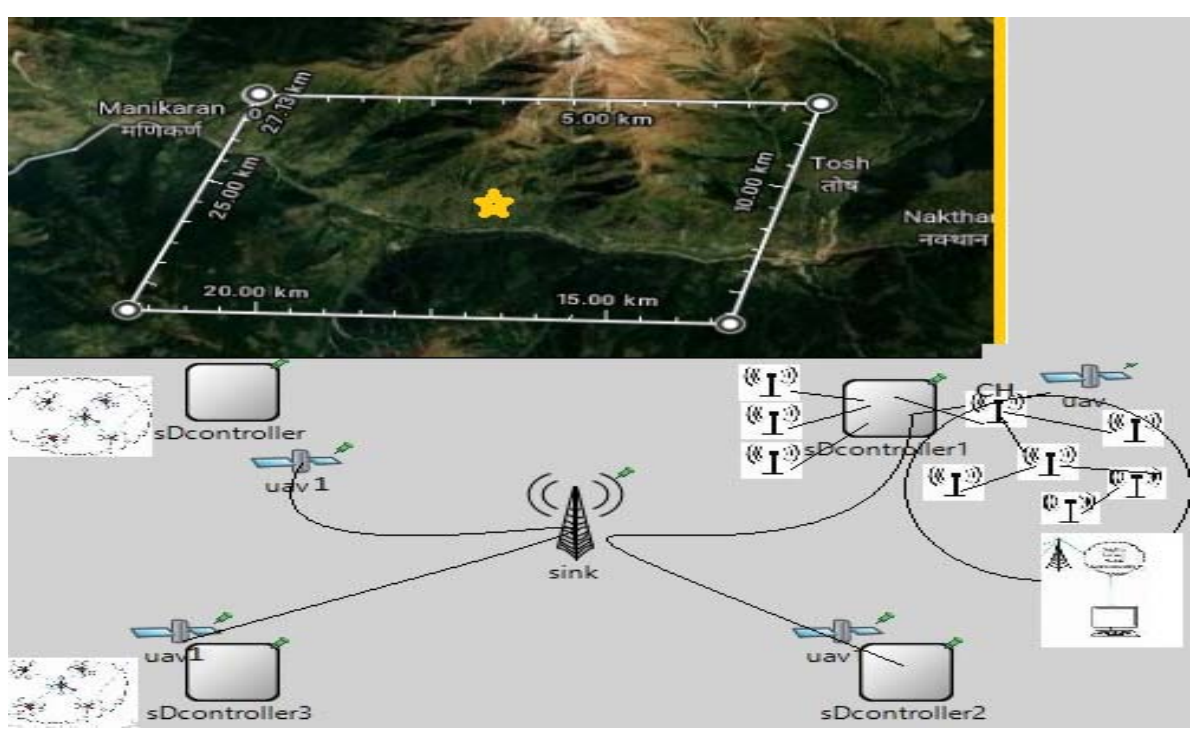

Figure1.12 UAV movement between the controller
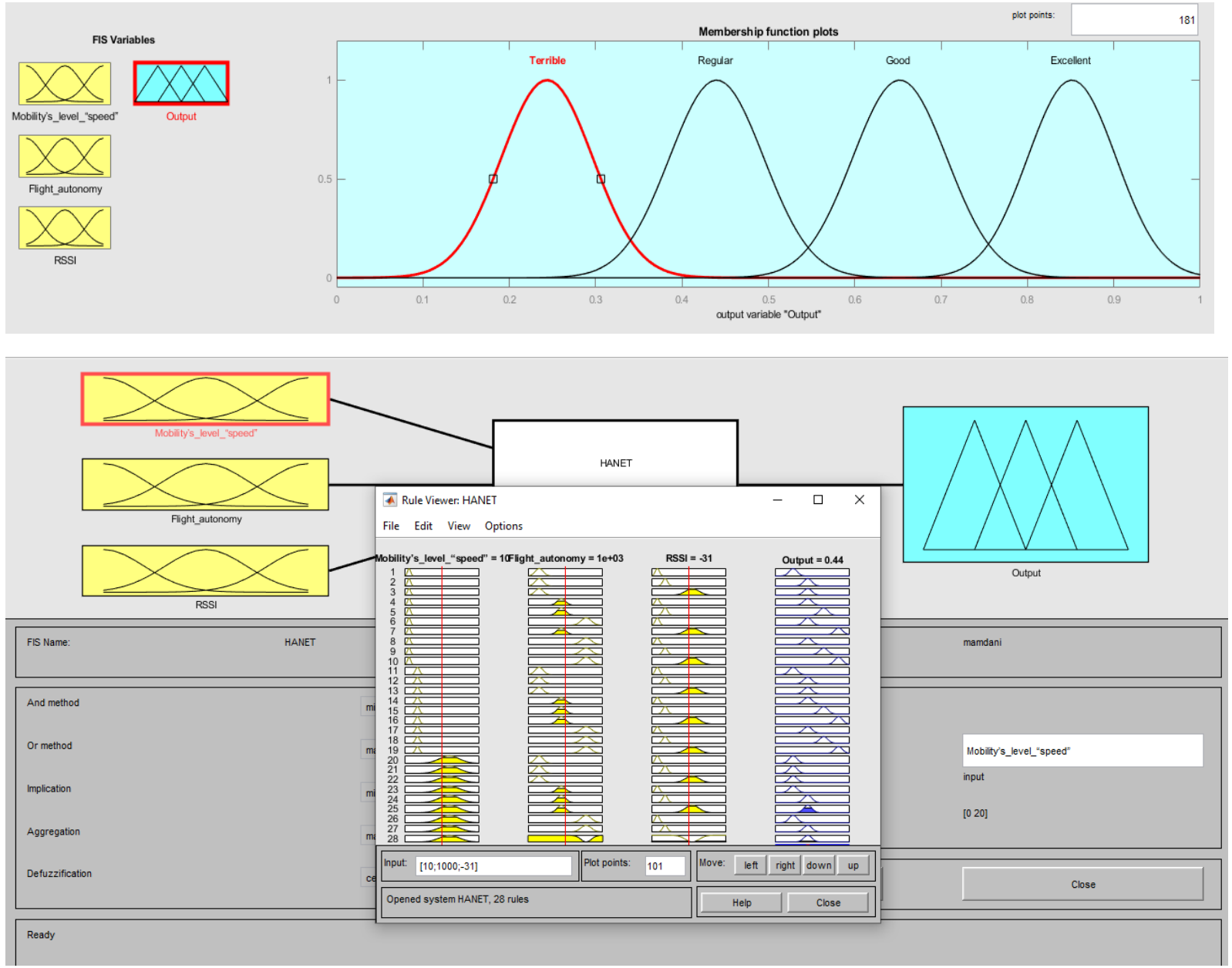

Figure1.13 Schematic view of proposal fuzzy rule-based system 


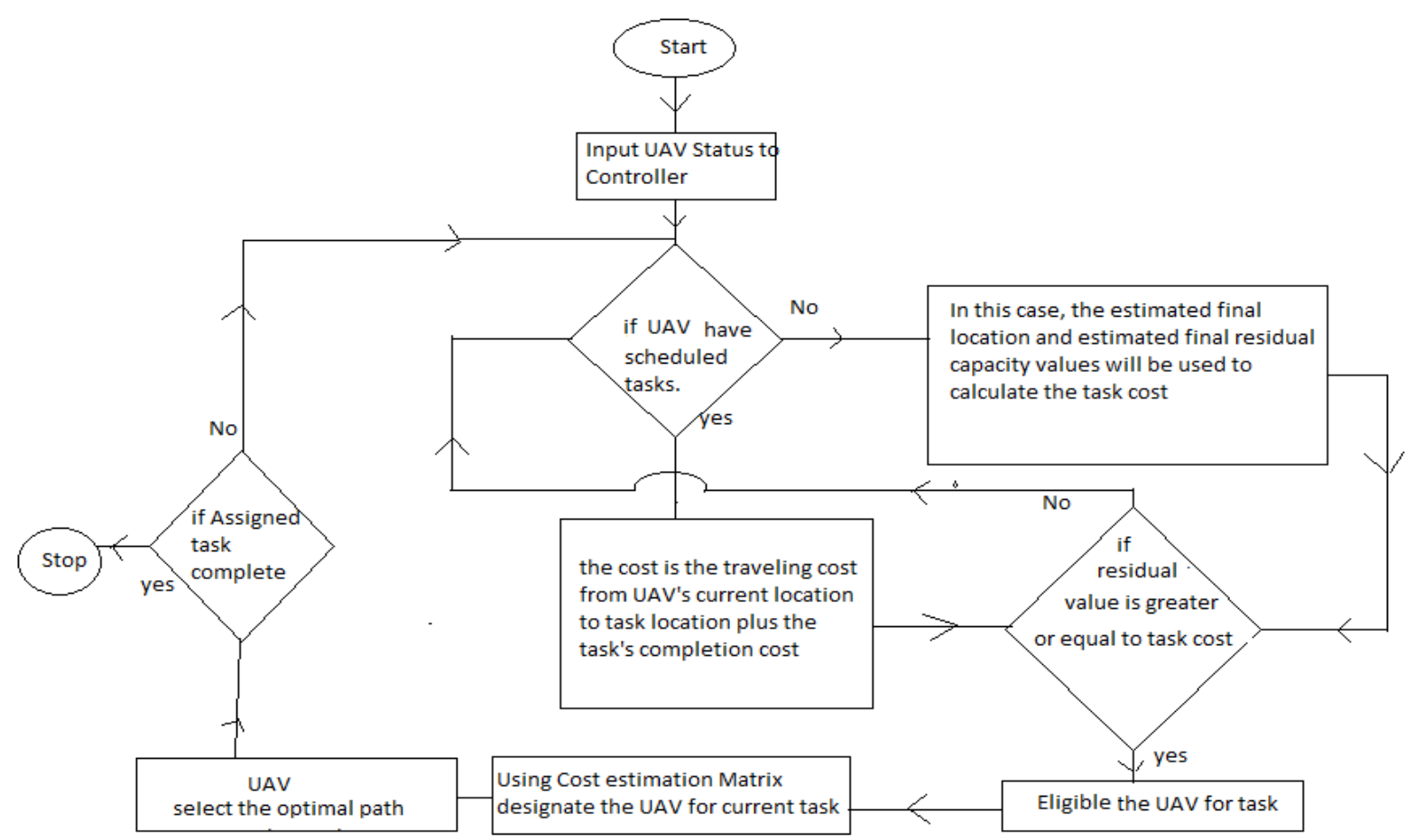

Figure1.14 UAV controller communication
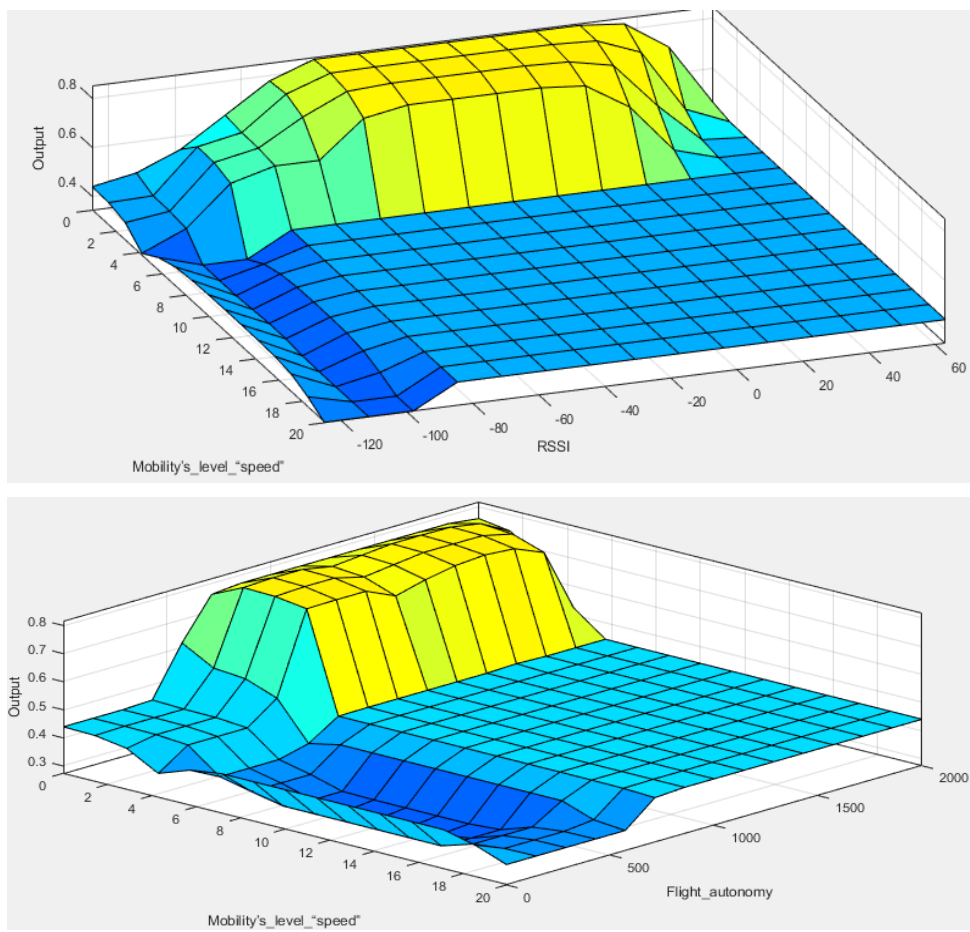

Figure1.15 3D view for the optimal and worst path

In the 3D images, the yellow portion proposed the most optimal path likely to be chosen since the UAV has a high RSSI linguistic value, low Mobility, and high flight autonomy. However, the green region represents the Average flight autonomy, RSSI, and Mobility. Simultaneously, the Blue region represents the highly dangerous path for the drone with low RSSI, high Mobility, and low flight autonomy. Due to the drone flying ability, it is possible to move closer and farther from each other, thus provide good communication as needed. Demography exhibits the comparison at varying timings. The horizontal axis shows the time at which the comparison is made, and the vertical axis shows the number of messages created in the network.

However, the user can also use Cbench[54], HCprobe, WCBench[46], OFCBenchmark, and OFNet[47] tools for evaluating the SDN controller[55] performance. 


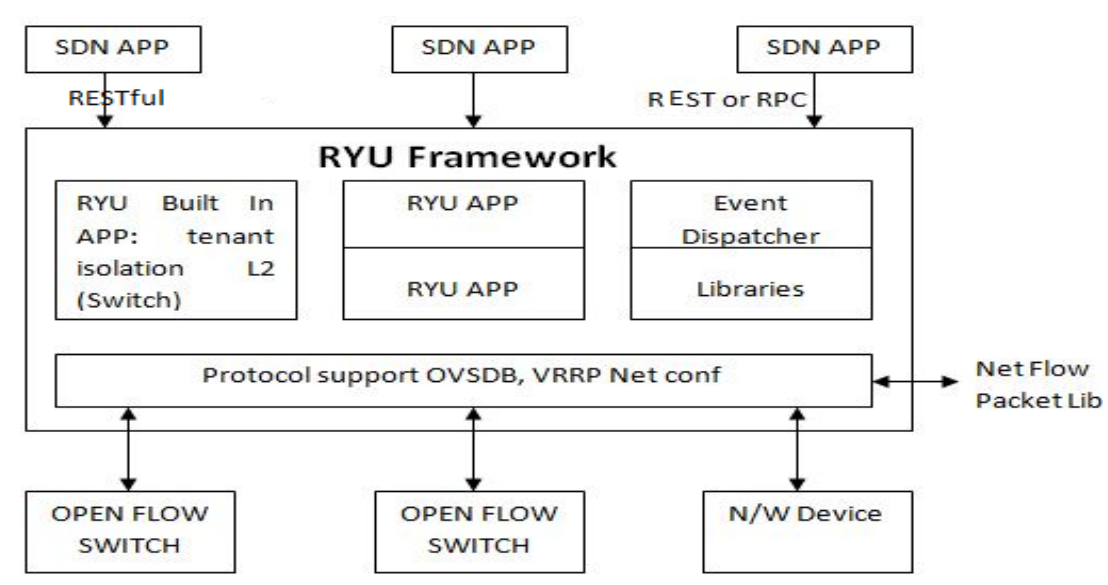

Figure1.16 RYU controller

\section{CONCLUSION}

In this paper, we have limelight the need for Software Defined Network for the future wireless network to redefine the principle communication. This is an agnostic approach for a holistic new concept in networking pursuit of an ad-hoc network; we defined it as HANET. This model plays a vital role in the development of the Adhoc network from many corners. The characteristics of the HANET network suggested being very good for large and mesh networks for enhancing the performance as compared to the traditional network. HANET network intends to incorporate extreme Mobility range, far away from reach, or hilly areas, Shows a unique way of connectivity on a single adaptation model. Moreover, the paradigm recipient that there is a continuous improvement in the performance. The algorithm divides the whole network model into the cluster and chooses the cluster Head for each cluster. The elected Custer Head measure the distance to get connects with its nearer SDN controller. Simulation results indicated that the increase in performance suggested high consistency and performance in the network for the future, as illustrated in figure 1.15, 3D view for the optimal and worst path, where the yellow path defined the most desired path for carrying data from SD controller to sink using UAV.

\section{ACKNOWLEDGMENT}

We thank the reviewers for their insightful comments. We sincerely indebted to their efforts and time.

\section{REFERENCES}

[1] R. Kirichek, A. Vladyko, A. Paramonov, and A. Koucheryavy, Software-Defined Architecture for Flying Ubiquitous Sensor Networking.

[2] Y. Qin, D. Huang, and B. Li, "STARS: A Statistical Traffic Pattern Discovery System for MANETs," IEEE Trans. Dependable Secur. Comput., vol. 11, no. 2, pp. 181-192, Mar. 2014, doi: 10.1109/TDSC.2013.33.

[3] Y. Zeng, Q. Wu, and R. Zhang, "Accessing from the Sky: A Tutorial on UAV Communications for 5G and beyond," Proc. IEEE, vol. 107, no. 12, pp. 2327-2375, 2019, doi: 10.1109/JPROC.2019.2952892.

[4] V. Dhiman, I. Saini, P. Manoj, M. Kumar, and P. Manoj, "A Comprehensive Survey of Location Based Routing in Vehicular Networks," I.J. Wirel. Microw. Technol., vol. 1, no. January, pp. 40-48, 2017, doi: 10.5815/ijwmt.2017.01.05.

[5] A. Pandey, P. K. Shukla, and R. Agrawal, "An adaptive Flying Ad-hoc Network (FANET) for disaster response operations to improve quality of service (QoS)," Mod. Phys. Lett. B, vol. 34, no. 10, pp. 1-25, 2020, doi: 10.1142/S0217984920500104.

[6] A. Chriki, H. Touati, H. Snoussi, and F. Kamoun, "FANET: Communication, mobility models and security issues," Comput. Networks, vol. 163, p. 106877, 2019, doi: 10.1016/j.comnet.2019.106877.

[7] Y. B. Ko and N. H. Vaidya, "Location-aided routing (LAR) in mobile ad hoc networks," Wirel. Networks, vol. 6, no. 4, pp. 307321, 2000, doi: 10.1023/A:1019106118419.

[8] H. Kim, J. Ben-Othman, and P. Bellavista, "Collision-free reinforced barriers in UAV networks," J. Comput. Sci., vol. 22, pp. 289-300, 2017, doi: 10.1016/j.jocs.2017.05.025.

[9] D. Orfanus, E. P. De Freitas, and F. Eliassen, "Self-Organization as a Supporting Paradigm for Military UAV Relay Networks," IEEE Commun. Lett., vol. 20, no. 4, pp. 804-807, 2016, doi: 10.1109/LCOMM.2016.2524405.

[10] B. Kate, J. Waterman, K. Dantu, and M. Welsh, "Simbeeotic," Proc. 11th Int. Conf. Inf. Process. Sens. Networks - IPSN '12, p. 49, 2012, doi: $10.1145 / 2185677.2185685$.

[11] Y. Wei, M. Brian Blake, and G. R. Madey, "An operation-time simulation framework for UAV swarm configuration and mission planning," Procedia Comput. Sci., vol. 18, pp. 1949-1958, 2013, doi: 10.1016/j.procs.2013.05.364.

[12] J. Guo, Y. He, and X. Zheng, "Pangu: Towards A Software-Defined Architecture for Multi-function Wireless Sensor Networks," doi: 10.1109/ICPADS.2017.00098.

[13] J. Souza, J. Jailton, T. Carvalho, J. Araújo, R. Francês, and Z. Kaleem, "A Proposal for Routing Protocol for FANET: A Fuzzy System Approach with QoE/QoS Guarantee," Wirel. Commun. Mob. Comput., vol. 2019, 2019, doi: 10.1155/2019/8709249.

[14] G. A. Kakamoukas, P. G. Sarigiannidis, and A. A. Economides, "FANETs in Agriculture - A routing protocol survey," Internet of Things, p. 100183, 2020, doi: 10.1016/j.iot.2020.100183.

[15] P. Jacquet, P. Mühlethaler, T. Clausen, A. Laouiti, A. Qayyum, and L. Viennot, "Optimized link state routing protocol for ad hoc networks," Proc. - IEEE International Multi Top. Conf. 2001 Technol. 21st Century, IEEE INMIC 2001, pp. 62-68, 2001, doi: 10.1109/INMIC.2001.995315.

[16] D. B. Johnson and D. A. Maltz, "Dynamic Source Routing in Ad Hoc Wireless Networks," Mob. Comput., pp. 153-181, 2007, 
doi: 10.1007/978-0-585-29603-6 5.

[17] P. Nayak, "Energy Aware Routing Scheme for Mobile Ad Hoc Network Using Variable Range Transmission," Int. J. Ad hoc, Sens. Ubiquitous Comput., vol. 3, no. 4, pp. 53-63, 2012, doi: 10.5121/ijasuc.2012.3406.

[18] T. Luo, H. P. Tan, and T. Q. S. Quek, "Sensor openflow: Enabling software-defined wireless sensor networks," IEEE Commun. Lett., vol. 16, no. 11, pp. 1896-1899, 2012, doi: 10.1109/LCOMM.2012.092812.121712.

[19] M. A. Araghizadeh, P. Teymoori, N. Yazdani, and S. Safari, "An efficient medium access control protocol for WSN-UAV," Ad Hoc Networks, vol. 52, pp. 146-159, 2016, doi: 10.1016/j.adhoc.2016.09.007.

[20] J. A. P. Fernández, L. J. G. Villalba, and T. H. Kim, "Software defined networks in wireless sensor architectures," Entropy, vol. 20, no. 4, pp. 1-23, 2018, doi: 10.3390/e20040225.

[21] S. Kaur, J. Singh, and N. S. Ghumman, "Network Programmability Using POX Controller," Int. Conf. Commun. Comput. Syst., p. 5, 2014, doi: 10.13140/RG.2.1.1950.6961.

[22] B. Nunes Astuto et al., "A Survey of Software-Defined Networking: Past, Present, and Future of Programmable Networks," vol. 16, no. 3, 2014, doi: 10.1109/SURV.2014.012214.00180ï.

[23] C. M. Cheng, P. H. Hsiao, H. T. Kung, and D. Vlah, "Maximizing throughput of UAV-relaying networks with the load-carry-anddeliver paradigm," IEEE Wirel. Commun. Netw. Conf. WCNC, pp. 4420-4427, 2007, doi: 10.1109/WCNC.2007.805.

[24] J. Alcaina, Á. Cuenca, J. Salt, M. Zheng, and M. Tomizuka, "Energy-efficient control for an unmanned ground vehicle in a wireless sensor network," J. Sensors, vol. 2019, 2019, doi: 10.1155/2019/7085915.

[25] O. K. Sahingoz, "Networking Models in Flying Ad-Hoc Networks ( FANETs ): Concepts and Challenges," 2013, doi: 10.1007/s10846-013-9959-7.

[26] A. I. Alshabtat and L. Dong, "Low latency routing algorithm for unmanned aerial vehicles ad-hoc networks," World Acad. Sci. Eng. Technol., vol. 80, no. 8, pp. 705-711, 2011.

[27] S. Rosati, K. Kruzelecki, L. Traynard, and B. Rimoldi, "Speed-aware routing for UAV ad-hoc networks," 2013 IEEE Globecom Work. GC Wkshps 2013, pp. 1367-1373, 2013, doi: 10.1109/GLOCOMW.2013.6825185.

[28] Y. Zheng, Y. Wang, Z. Li, L. Dong, Y. Jiang, and H. Zhang, "A mobility and load aware OLSR routing protocol for UAV mobile AD-HOC networks," 2014 Int. Conf. Inf. Commun. Technol. ICT 2014, 2014.

[29] M. K. Denko and J. Tian, "Cross-layer design for cooperative caching in mobile ad hoc networks," 2008 5th IEEE Consum. Commun. Netw. Conf. CCNC 2008, pp. 375-380, 2008, doi: 10.1109/ccnc08.2007.90.

[30] V. Dhiman, "BIO Inspired Hybrid Routing Protocol for Wireless Sensor Networks," Www.Ijaret.Org, vol. 1, no. Iv, pp. 33-36, 2013.

[31] J. H. Forsmann, R. E. Hiromoto, and J. Svoboda, "A time-slotted on-demand routing protocol for mobile ad hoc unmanned vehicle systems," Unmanned Syst. Technol. IX, vol. 6561, p. 65611P, 2007, doi: 10.1117/12.719355.

[32] R. Shirani, Reactive-Greedy-Reactive in Unmanned Aeronautical Ad-hoc Networks: A Combinational Routing Mechanism, vol. MR83052, no. August. 2011.

[33] V. D. Park and M. S. Corson, "Temporally-Ordered Routing Algorithm," J. Chem. Inf. Model., vol. 53, no. 9, pp. 1689-1699, 2019, doi: 10.1017/CBO9781107415324.004.

[34] B. Karp and H. T. Kung, "GPSR: Greedy Perimeter Stateless Routing for wireless networks," Proc. Annu. Int. Conf. Mob. Comput. Networking, MOBICOM, no. MobiCom, pp. 243-254, 2000.

[35] R. L. Lidowski, B. E. Mullins, and R. O. Baldwin, "A novel communications protocol using geographic routing for swarming UAVs performing a search mission," 7th Annu. IEEE Int. Conf. Pervasive Comput. Commun. PerCom 2009, 2009, doi: 10.1109/PERCOM.2009.4912764.

[36] D. Medina, F. Hoffmann, F. Rossetto, and C. H. Rokitansky, "North atlantic inflight internet connectivity via airborne mesh networking," IEEE Veh. Technol. Conf., 2011, doi: 10.1109/VETECF.2011.6092878.

[37] L. Lin, Q. Sun, J. Li, and F. Yang, "A novel geographic position mobility oriented routing strategy for UAVs," J. Comput. Inf. Syst., vol. 8, no. 2, pp. 709-716, 2012.

[38] S. Venkatraman and S. K. Sarvepalli, "Load balance technique with adaptive position updates (LAPU) for geographic routing in MANETs," Eurasip J. Wirel. Commun. Netw., vol. 2018, no. 1, 2018, doi: 10.1186/s13638-018-1072-y.

[39] S. Wang, C. Fan, C. Deng, W. Gu, Q. Sun, and F. Yang, "A-GR: A novel geographical routing protocol for AANETs," J. Syst. Archit., vol. 59, no. 10 PART B, pp. 931-937, 2013, doi: 10.1016/j.sysarc.2013.07.011.

[40] K. Saifullah and K. Il Kim, "A new geographical routing protocol for heterogeneous aircraft Ad Hoc Networks," AIAA/IEEE Digit. Avion. Syst. Conf. - Proc., pp. 1-9, 2012, doi: 10.1109/DASC.2012.6382336.

[41] N. Gude, J. Pettit, B. Pfaff, N. Mckeown, and S. Shenker, "Nox-Ccr-Final.Pdf."

[42] Z. Cai, A. Cox, and E. T. S. Ng, "Maestro: A System for Scalable OpenFlow Control," Cs.Rice.Edu, p. 10, 2011, [Online]. Available: http://www.cs.rice.edu/ eugeneng/papers/TR10-11.pdf.

[43] D. Erickson, "The Beacon OpenFlow controller," HotSDN 2013 - Proc. 2013 ACM SIGCOMM Work. Hot Top. Softw. Defin. Netw., pp. 13-18, 2013, doi: 10.1145/2491185.2491189.

[44] I. Z. Bholebawa and U. D. Dalal, "Performance analysis of SDN/openflow controllers: POX versus floodlight," Wirel. Pers. Commun., vol. 98, no. 2, pp. 1679-1699, 2018, doi: 10.1007/s11277-017-4939-z.

[45] P. Berde et al., "ONOS: Towards an open, distributed SDN OS," HotSDN 2014 - Proc. ACM SIGCOMM 2014 Work. Hot Top. Softw. Defin. Netw., pp. 1-6, 2014, doi: 10.1145/2620728.2620744.

[46] S. Asadollahi, B. Goswami, and A. M. Gonsai, "Implementation of SDN using OpenDayLight Controller," vol. 5, no. 2, pp. 218 227, 2017.

[47] "Modular SDN Programming with Pyretic."

[48] A. Voellmy, H. Kim, G. Tech, and N. Feamster, Procera: A Language for High-Level Reactive Network Control, vol. 12. 2012.

[49] S. Asadollahi and M. Sameer, "Ryu Controller's Scalability Experiment on Software Defined Networks," pp. 2-6.

[50] M. T. Islam, N. Islam, and M. Al Refat, "Node to Node Performance Evaluation through RYU SDN Controller," Wirel. Pers. Commun., vol. 112, no. 1, pp. 555-570, 2020, doi: 10.1007/s11277-020-07060-4.

[51] M. Tiloca, A. Stagkopoulou, and G. Dini, "Performance and Security Evaluation of SDN Networks in OMNeT++/INET," 2016, [Online]. Available: http://arxiv.org/abs/1609.04554.

[52] B. Lantz and B. O'Connor, "A Mininet-based Virtual Testbed for Distributed SDN Development," ACM SIGCOMM Comput. Commun. Rev., vol. 45, no. 5, pp. 365-366, 2015, doi: 10.1145/2829988.2790030.

[53] A. T. Albu-salih, "Dynamic Routing Method over Hybrid SDN for Flying Ad Hoc Networks Abstract :," vol. 15, no. 3, pp. 361$368,2018$.

[54] A. Sonba and H. Abdalkreim, "Performance Comparison Of the state of the art Openflow Controllers," pp. 1-60, 2014, [Online]. Available: http://www.diva-portal.org/smash/get/diva2:783679/FULLTEXT02.

[55] L. Zhu, K. Sharif, F. Li, X. Du, and M. Guizani, "SDN Controllers : Benchmarking \& Performance Evaluation," pp. 1-14. 


\section{AUTHOR PROFILE}
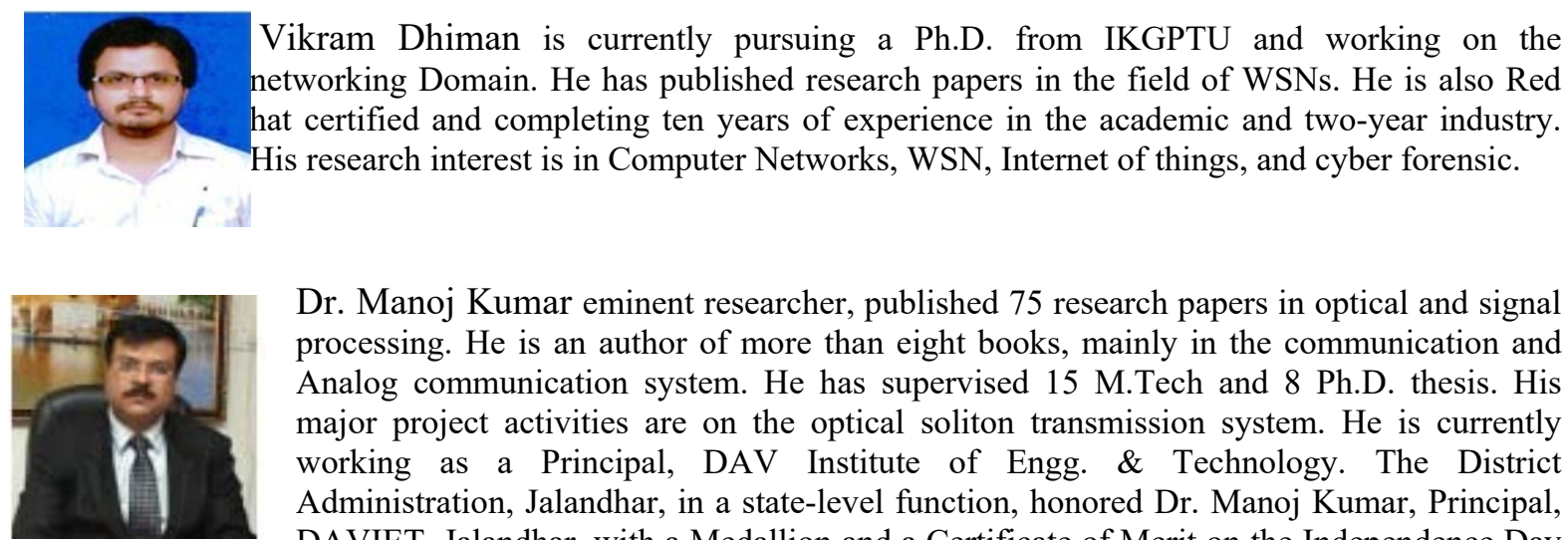

Dr. Manoj Kumar eminent researcher, published 75 research papers in optical and signal processing. He is an author of more than eight books, mainly in the communication and Analog communication system. He has supervised 15 M.Tech and 8 Ph.D. thesis. His major project activities are on the optical soliton transmission system. He is currently working as a Principal, DAV Institute of Engg. \& Technology. The District Administration, Jalandhar, in a state-level function, honored Dr. Manoj Kumar, Principal, DAVIET, Jalandhar, with a Medallion and a Certificate of Merit on the Independence Day 2018 recognizing his services for "outstanding contribution to education and various government programs." Dr. Manoj Kumar has been honored with "LMA -Dayanand Munjal Award for Manager of the year 2017", the most prestigious annual award instituted by LMA since 1984 \& sponsored by Hero Cycles Limited, Ludhiana, for his outstanding Innovative and Leadership Achievements. Dr. Manoj Kumar has selected under AICTE-UKIERI Technical Leadership Development Program supported by the British Council for the AY 2018-19. He received the "Jewel of India" award by the Indian Solidarity Council for 2006, Silver Medal in M.Tech. (Electronics \& Comm. Engg.) From Punjab Technical University, Jalandhar, Best PI coordinator award from Secretary, DOE, and Govt. of India under the IMPACT-SSS project sponsored by DOE, GOI; World Bank, and Swiss Development Cooperation, Switzerland. He is a Reviewer for Elsevier Science's International Journal-Optical Fiber Technology, Springer, ICFAI Journals, and World Scientific \& Engineering Academy and Society (WSEAS) for international conferences. He is a member of the FICCI North Region Task Force on Higher Education.

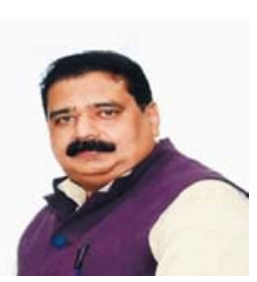

Dr. Ajay K. Sharma Joined IKG Punjab Technical University, Jalandhar, as 13th ViceChancellor on March 27 2018. Earlier, Prof. Sharma was Director, National Institute of Technology, Delhi from October 10, 2013, to March 26 2018, and during this period, he also served as Director, Hamirpur HP (Additional Charge) from May 3, 2016, to March 21, 2018, and Mentor Director (Additional Charge), IIIT Una from May 3, 2016, to March 21, 2018. Needless to mention that during the above tenures of Director and Vice-Chancellor, he contributed to the several academic and administrative reforms to the system of Higher Education. He received his BE in Electronics and Electrical Communication Engineering from Panjab University Chandigarh, India in 1986, MS in Electronics and Control from Birla Institute of Technology (BITS), Pilani in the year 1994, and Ph.D. in Electronics Communication and Computer Engineering in the year 1999 from NIT Kurukshetra (Erstwhile Regional Engineering College). His Ph.D. thesis was on "Studies on Broadband Optical Communication Systems and Networks." After serving various organizations from 1986 to 1995, he has joined the National Institute of Technology (Erstwhile Regional Engineering College) Jalandhar as Assistant Professor in the Department of Electronics and Communication Engineering in the year 1996. From November 2001, he has worked as Professor in the ECE department, and thereafter he has worked as Professor in Computer Science \& Engineering from 2007 to 2013 in the same institute. His primary areas of interest are broadband optical wireless communication systems and networks, dispersion compensation, fiber nonlinearities, optical soliton transmission, WDM systems and networks, Radioover-Fiber (RoF), and wireless sensor networks and computer communication. He has published 342 research papers in the International/National Journals/Conferences and 12 books. He has supervised $30 \mathrm{Ph} . \mathrm{D}$. and 46 M.Tech Thesis. He has completed four R\&D projects funded by the Government of India, and one project is ongoing. He was associated with implementing the World Bank project of 209 Million for the TEQIP-I program of the institute. He is President's/Visitor's Nominee, All NITs for Engineering and Technology Group, w.e.f. May 19, 2017, to May 18 2020. Sri Guru Nanak Dev Ji Achievers Award: In Recognition of my valuable contribution to education, The Government of Punjab conferred Sri Guru Nanak Dev Ji Achievers Award on the auspicious occasion of the 550th Birthday of Sri Guru Nanak Dev Ji on November 10 2019, at IKGPTU, Kapurthala, Punjab. He was conferred the Honorary Fellowship Award by the Punjab Academy of Sciences during the 22nd Punjab Science Congress on February 7, 2019, for Recognition of his outstanding research contributions and achievements in Engineering Sciences. He has been awarded Shane-e-Hind for Recognition and contributions in Technical Education by the Sarv Kalaynkari Society. 